\title{
Extending Vision: Sky-situated Knowledge and the Artist's Eye
}

\section{Felicity Spear}

\begin{abstract}
Increasingly, we are looking beyond our planet to speculate about our place in the Universe. In the context of ideas about the sky and cosmic space, art works have the potential to provoke curiosity and to play an educative and imaginative role in visualising connections with science, history and a space beyond the full range of our senses. While both artists and scientists reconstruct the material world on the basis of understanding, artists are able to exploit subjectivity and are not accountable to demonstrate proof. In this way, art seeks a poetic dimension or insight which speaks of things outside art in new or different ways. This paper discusses my recent research and the exhibitions I curated in order to coincide with the 2009 International Year of Astronomy. Included in these were an astro-photographer and a number of Australian artists, both Western and Indigenous, whose work has been influenced by the speculative and experimental processes involved with observation, image-capture and mapping, and the technological developments which shape human consciousness. They draw also on the history of human efforts to picture whatever lies beyond Earth's atmosphere. This space, mostly beyond the naked eye, is revealed now through a machine-produced visibility which extends our vision. Together, these works show us how various systems of knowledge have sought to make sense of the cosmos and our place within it.
\end{abstract}

\section{Extending vision: sky situated knowledge and the artist's eye}

What happens when artists and scientists share a field of inquiry? It is possible that, while exploring fundamental structures of the material world, scientists and artists are able to uncover overlapping insights about the nature of reality. In recent times our thinking has been increasingly determined through a set of interacting processes and relational fields that are more spontaneous and less rule-bound. It is in this context that I suggest new conversations between art and science might be possible. Although, as we know, art and science are not the same thing, both pursuits are driven by imagination and curiosity. Science is generally regarded as our most reliable knowledge system for understanding the physical world. Artists however, while interpreting or recreating nature on the basis of understanding, are not accountable to demonstrate proof.

Felicity Spear, 'Extending Vision: Sky-situated Knowledge and the Artist's Eye', eds. Nicholas Campion and Rolf Sinclair, Culture and Cosmos, Vol. 16 nos. 1 and 2, 2012, pp. 451-463.

www.CultureAndCosmos.org 
The artist searches for a poetic dimension or insight which allows for subjectivity and speaks of things outside art in new ways.

Increasingly we are looking beyond our planet to speculate about our place in the Universe. From a cosmic perspective, science tells us that Earth is a totally insignificant speck of dust in an expanding and accelerating Universe that may eventually disappear. As the cosmologist John Barrow observes, 'we feel like the Universe's only child, and that feeling has many consequences'. ${ }^{1}$ It raises questions. When we look into the night sky, how many of us are aware that we are seeing beyond our genetic programming, looking through time into a remote past? The night sky is a largely hidden extension of the natural world. When culture and its technologies are laid over nature like a map, what are the implications for our sense of the real? Does the creation of a synthetic nature stimulate our desire to seek out 'real' nature? Over time and with new technologies and digital processes, the dividing line between out there and back here is becoming increasingly blurred, changing our understanding of time and space. Every there now seems also to be here.

Although the ineluctable fact remains that our bodies are our referents, direct experience cannot account for phenomena beyond the visible. What is required of the artist or the scientist is the creation of models or mental constructs which, sometimes in an abstract way, are able to become projections across space and time. The artist and writer Vik Muniz says of the model:

[i]t marks the turning point between mind and matter. While artists create models that expand our picture of the world beyond its physical reality, scientists make models that expand our understanding of the physical world beyond the limits of perception. In both cases a mental image is tested against material - but while the artist adapts matter to his imagination, the scientist adapts his imagination to what he discovers about matter. $^{2}$

With this in mind, my paper will discuss an exhibition, which emerged from my recent research, titled Beyond visibility: light and dust (Figure 1). This brought together my own work, the work of an astronomer and

\footnotetext{
${ }^{1}$ John D. Barrow, The Artful Universe, (Oxford: Clarendon Press, 1995), p. 44.

${ }^{2}$ Vic Muniz, Reflex A Vik Muniz Primer (New York: Aperture Foundation, 2005), p. 177.
} 
scientific photographer, David Malin, and that of an indigenous Australian Aboriginal artist, Gulumbu Yunupingu. The exhibition was planned to coincide with the 2009 International Year of Astronomy and took place last year in Melbourne and Sydney. Each of us has created work which focuses on perception beyond visibility. It was developed to raise awareness of the resonances to be found within various systems of knowledge, focusing on the cosmos and our understandings of our place within it.

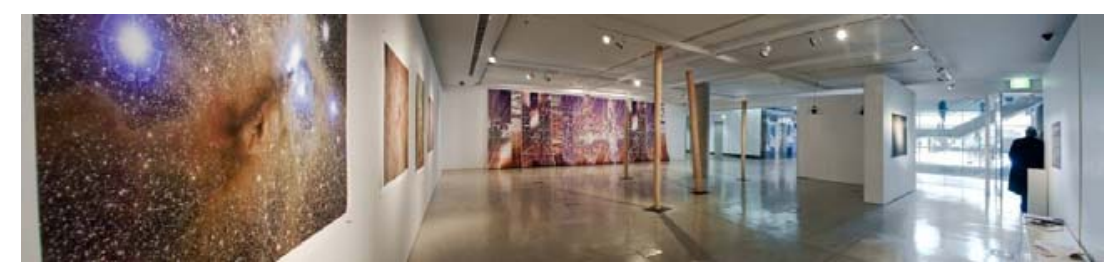

Figure 1. David Malin, Felicity Spear, Gulumbu Yunupingu Beyond visibility: light and dust, 2009, mixed media, University of Technology Sydney Gallery (Photo Paul Pavlou)

To accompany the Sydney exhibition, (and also the exhibition Shared Sky, mounted in 2009 by the National Gallery of Victoria), I created a sound installation titled Out There: in light of remote possibilities. It is constructed from a selection of star radio-frequency samples, emitted at a wide range of frequencies, in many different regions of deep space. This data was made available through Mt. Stromlo Observatory, the Australian National University, and the Jodrell Bank Observatory. Through a nonvisual sense, the ambient sound data had an integral relationship to the other works in the gallery.

Australian Aboriginal culture is the oldest continuous culture in the world. It is likely therefore that the Australian Aboriginal people would have been the world's first astronomers, mapping the night sky in their own idiosyncratic ways with a variety of representations and symbolic forms which hover between subjective and objective reality. Gulumbu Yunupingu, now in her sixth decade, is a member of the indigenous Yolgnu people of North East Arnhem Land, situated on the edge of Australia's Gulf of Carpentaria. With a humanist eye, she focuses her meditations about the Universe on the links between people on earth and the night sky.

In her catalogue essay for Beyond visibility: light and dust, the art historian Elina Spilia relates that, in the 1960s, Gulumbu's father was 
witness to the building of a satellite tracking station in the eucalypt forests of Gulkula, on his people's ancestral land. The station was established by the European Launcher Development Organization. It is believed by the Yolgnu that this place is inhabited by the ghostly spirit of Ganbalapula who, while wandering in this territory, turned his gaze upward to seek honey from the hollows in the trees, metaphorically seeking the sweetness of knowledge. The word dhupuma, translated as the elevated gaze or the elevated mind, is part of the Yolgnu language and tradition. This word dhupuma could be seen as a metaphor which links different systems of knowledge through the observation of a shared sky. Our evolution is mapped in these webs of connections, between out there and back here.

For indigenous Australians, the Earth is reflected symbolically in the sky. Aboriginal cosmologies identify certain celestial bodies, as well as the spaces of darkness in between them, to be significant precursors of ancestral knowledge, the evidence of which is then embedded in sacred objects. Just as the darkness in the sky represents knowledge beyond the visible, these objects, often protected from public view, represent revelations of ancestral law. Spilia explains further:

The idea that meaning exists beyond visible perception is a grounding principle of ancestral revelation, and one that is transposed into Yolgnu aesthetics. According to Yolgnu scholarship, an invisible or semi-visible trace may indicate an imminent but obscured presence, power or thing that holds profound and secreted meaning. Astrophysicists provide us with an apt analogy: celestial bodies are not perceptible in themselves: they are visible only through fragments of light beamed across unfathomable light years: eye and light join across distance and time to provide traces of an ancient past manifested in the present. ${ }^{3}$

For this exhibition, Gulumbu has constructed bark paintings and larrakitj or memorial poles, titled Garak - the Universe. As we wander through this forest of hollow logs, wrapped around with subtly layered variations of patterned star forms and dot-like particles of paint, it seems as if we

\footnotetext{
${ }^{3}$ Elina Spilia, 'Dhupuma' in S. Lakin (ed.), Beyond visibility: light and dust, exhibition catalogue (Melbourne: Monash Gallery of Art), p. 5.
} 
are immersed in the wide expanse of the night sky as in a forest of stars and galaxies.

The large stars are those visible to the naked eye, and the dots represent what remains invisible (Figures 2 and 3). Like an x-ray view, these intricately detailed surfaces painted in natural pigments suggest the invisible depths of the Universe and the metaphorical mapping of the cycles and atomistic interconnectedness of all life. Gulumbu's paintings emphasise both the universal and the culturally specific. For Western eyes, they suggest subliminal mathematical relationships that link art to science, metaphysics and epistemology. She speaks in a both humble and philosophic way about the ideas she hopes the viewer will find in her work: 'When you look up, go deeper until you can see the dots in my painting. Look around, keep studying. The mind goes up and up, we don't know where or how deep it goes'. ${ }^{4}$

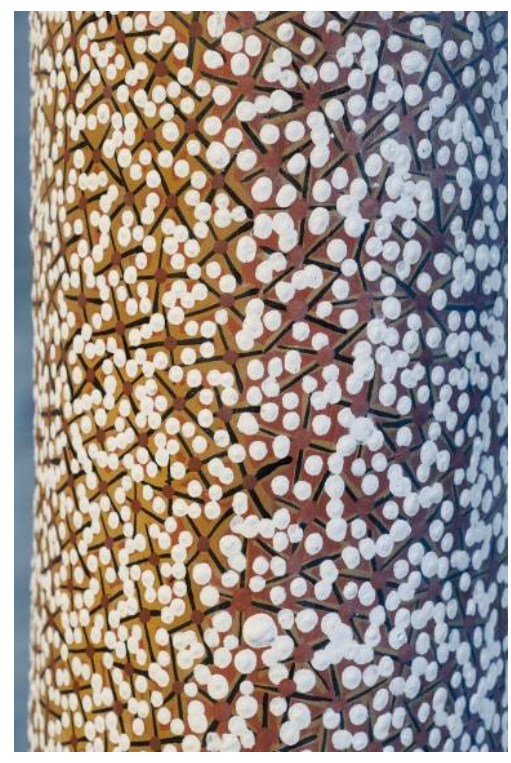

Figure 2. Gulumbu Yunupingu, Garak the Universe, 2008 (detail), natural pigments on wood

\footnotetext{
${ }^{4}$ Gulumbu Yunupingu, in 'Dhupuma' in S. Lakin (ed.), Beyond visibility: light and dust, exhibition catalogue (Melbourne: Monash Gallery of Art), p.15.
}

Culture and Cosmos 
456 Extending Vision: Sky-situated Knowledge and the Artist’s Eye

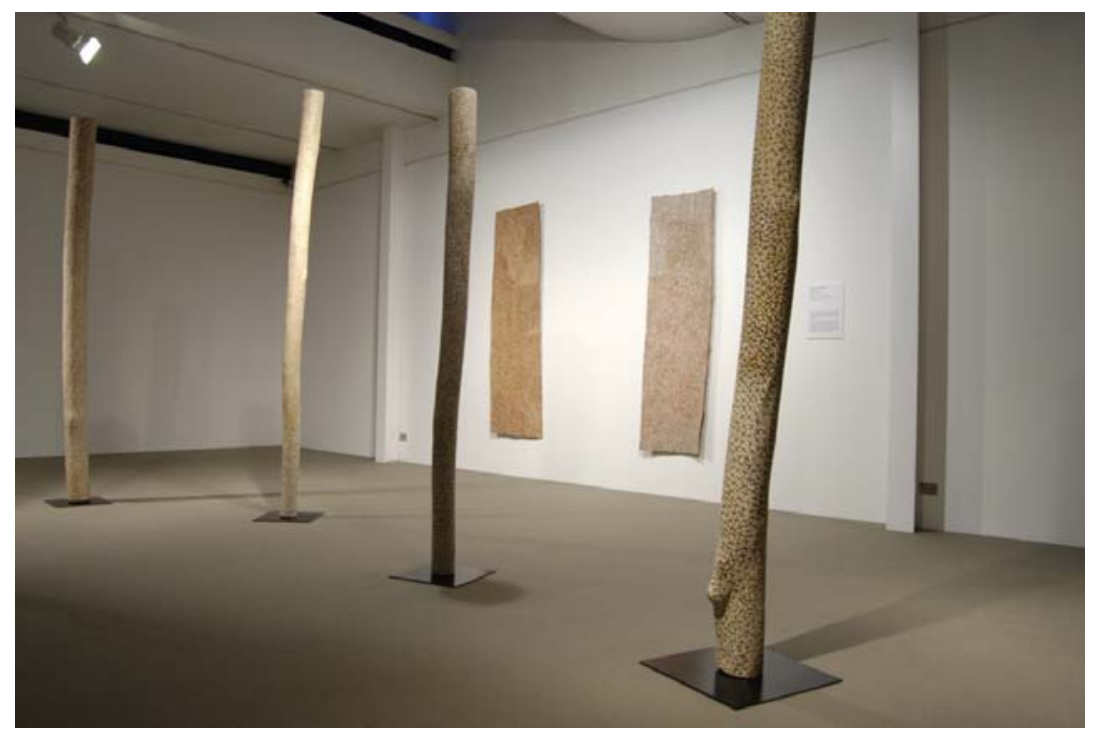

Figure 3. Gulumbu Yunupingu, Garak - the Universe, 2008, natural pigments on bark and wood, Monash Gallery of Art Melbourne

While working as a photographic scientist at the Anglo-Australian Observatory, David Malin developed innovative imaging processes which enabled considerable enhancement of celestial bodies, most of which represent phenomena beyond the human eye. These pioneering analogue photographs captured the first true-colour images of deep space. The human eye is unable to see colours when light levels are low, so light is enhanced through colour to make such phenomena visible. Taken with a prime focus camera mounted in a cage at the Observatory, David explains the process:

[they are taken] with three black and white exposures through separate coloured filters of red, green and blue. These are then superimposed onto photographic film, resulting in images of stars with their actual colours, thus giving an indication of their age, size, temperature and evolutionary history....[the] allotment of precious telescope time [has been] usually 5-8 nights a year, and sometimes some of those nights are cloudy. ${ }^{5}$ 5 The Man Who Colours Stars, pp. 1-2, http://hoa.aavso.org/postermalin.htm,
[accessed 25 Aug 2006].

Culture and Cosmos 
In twenty years David has captured fewer than 200 pictures, some of which have taken years to produce. In this exhibition he exhibits six large-scale inkjet prints of his astronomy photographs. They are celestial phenomena found in the southern sky, the Australian sky. These are images which map the vast multitudes of starfields in the Milky Way and, between them, dark clouds of dust like particles of sand on a beach, from which we and the stars are made. They were selected not only for their relationship to our own Milky Way galaxy, but also because, when seen in the context of Gulumbu's work Garak, and my own work Deep Field, they create links philosophically, intellectually and aesthetically (Figure 4).

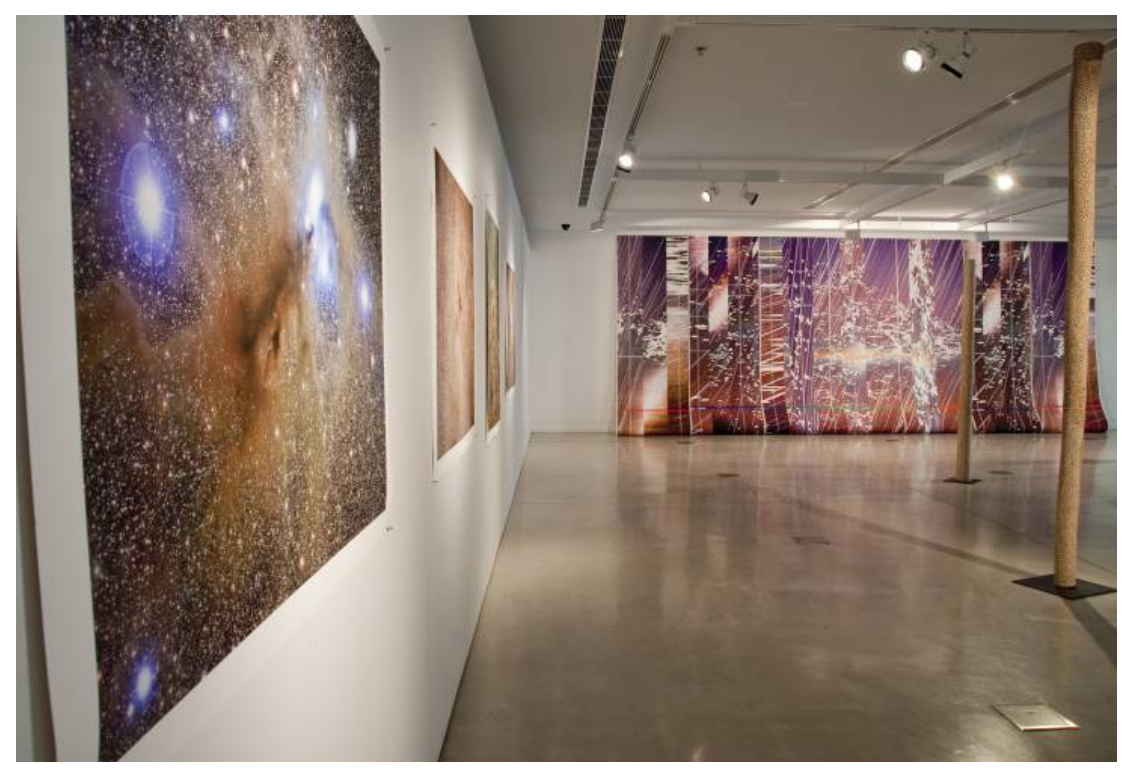

Figure 4. David Malin, Felicity Spear, and Gulumbu Yunupingu, Beyond visibility: light and dust, 2009, mixed media, University of Technology Sydney Gallery (Photo Paul Pavlou)

The six images under discussion here can be found at www.davidmalin.com/. Of the image Corona Australis reflection nebula, with its globular cluster NGC 6723 (Figure 5), 30,000 light years distant in Sagittarius, David points out that, 'almost all the nebulosity in the image is starlight, reflected from minute grains of dust, some of which gather into darker condensations, 'molecular clouds' blotting out the background stars while the brighter ones are embedded in it'. 
458 Extending Vision: Sky-situated Knowledge and the Artist’s Eye

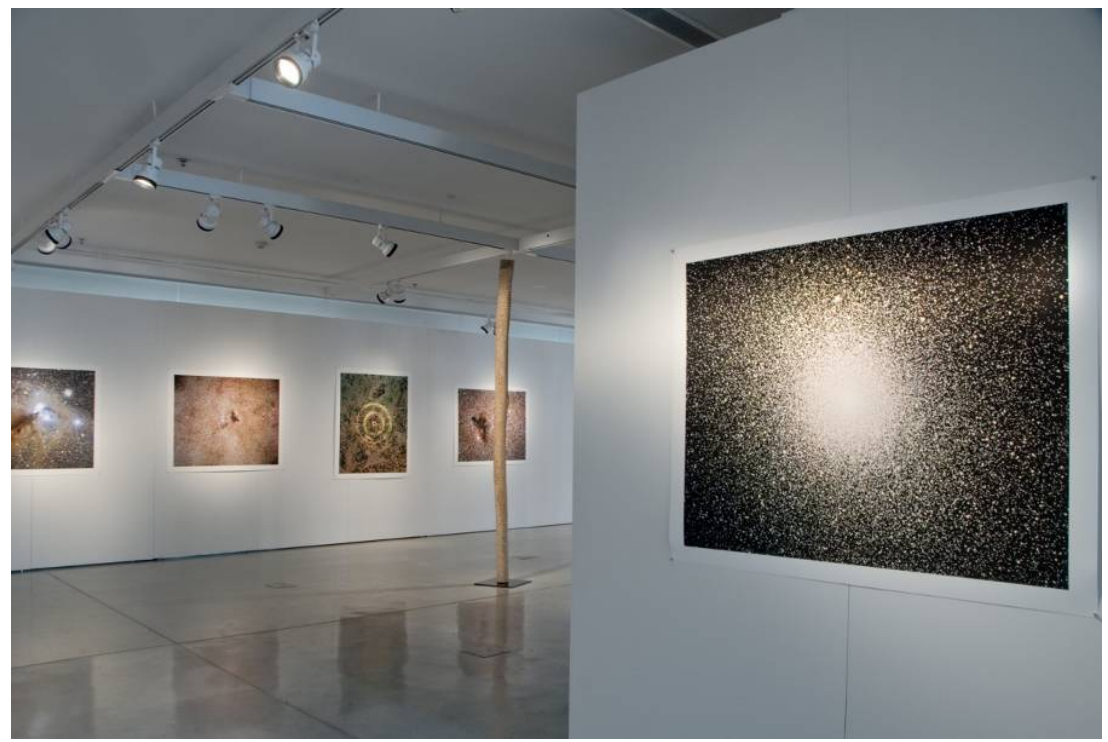

Figure 5. David Malin, uks037a Corona Australis reflection nebula, 2008, pigment inkjet print

David describes the Globular Cluster 47 Tucanae 2007 (aat 076) (Figure 6), as an ancient city of stars, a faded remnant of energetic bursts of star formation 15,000 light years distant. In the Clouds of stars and dust in Sagittarius 2008 (uks 020), he points out myriad ancient stars crisscrossed with dusty streaks and dark clouds pricked with star clusters. The Wide field view of Barnard 86 and NGC 65202007 (aat 092), is described as being cool, yellow ancient star fields of the southern Milky Way orbiting the inner part of the galaxy, and the clusters of younger, mainly blue stars NG 6520 are found in the spiral arms. A closer view of the region Dust cloud and open cluster NGC 65202006 (aat022), reveals the dark Cloud Barnard 86 and the cluster of hotter, bright blue stars. Finally, in the image, the Light echo of supernova 1987A, David has captured a self-destructing star in the large Magellanic Cloud close by our Milky Way galaxy. It exploded in a brilliant flash of light visible to the naked eye. Some light was deflected by two sheets of dust near the star. These light echoes, recorded in a series of six images, (the last being about five years after the appearance of the supernova), appear to expand with time. This light has taken 170,000 light years to reach us and was at its brightest in May 1987. 


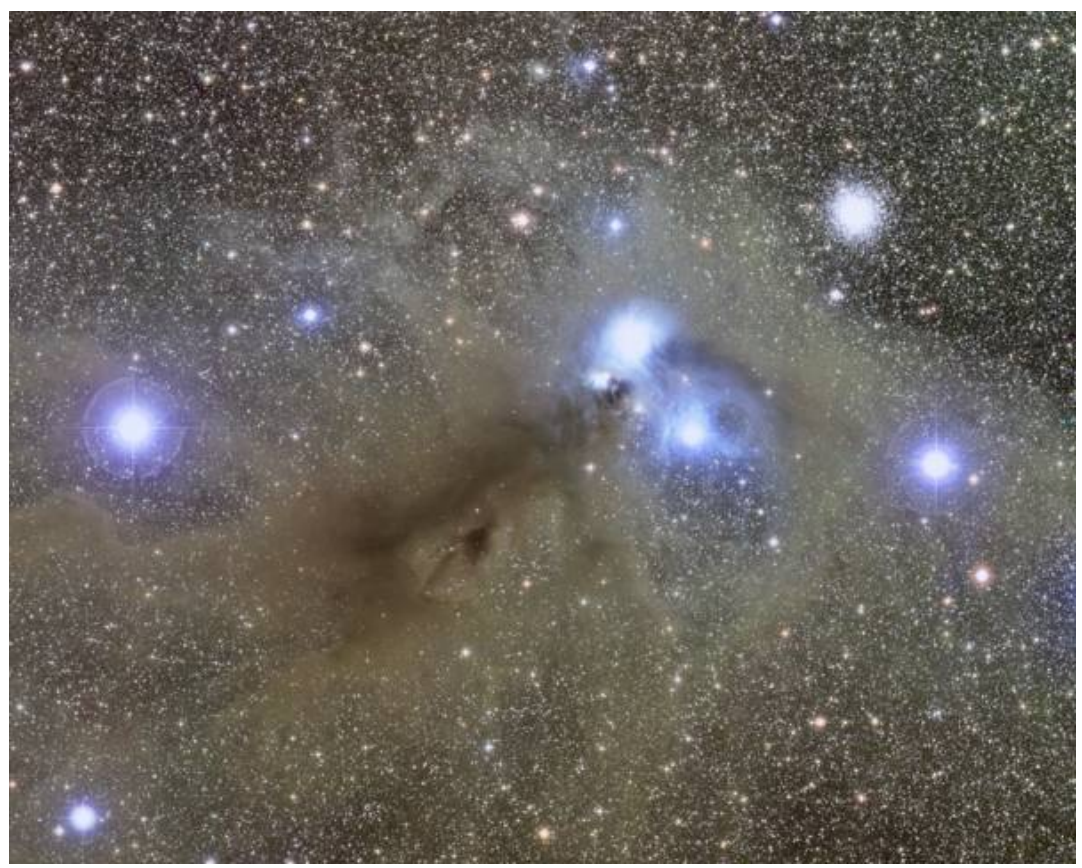

Figure 6. David Malin and Gulumbu Yunupingu, Beyond visibility: light and dust, 2009, mixed media, University of Technology Sydney Gallery (Photo Paul Pavlou)

These images demonstrate that photography has, in more recent times, detected and mapped an unseen and un-seeable Universe. The light captured on David's photographic plates has travelled through space and time, sometimes for millions of years. When we look into these images we are looking at things as they were then, not as they are now. Such images represent both the intellectual and artistic dimension of mapping. The map historian Peter Whitfield has noted that, 'the taste for visual drama which was once satisfied by the mythology of the constellations has been transferred from maps to photographs'. ${ }^{6}$

My recent works are based on the concept of mapping as it might be understood as a creative and experimental practice, a process of deconstructing and reassembling selected components, a way of thinking about and organizing space. As technology stands in for first-hand sense experience, so mapping stands in for space, giving a form of visible

${ }^{6}$ Peter Whitfield, Mapping The Heavens (London: The British Library, 1995), p. 128.

Culture and Cosmos 
reality (the map), to an invisible reality (the space being mapped). Every shift in technology has required us to rethink our ideas about what is meant by reality.

As a painter and print maker my work increasingly resembles a data-saturated surface. Visual art has, for a long period, been engaged with optical and mechanical devices such as the camera obscura, later photography and now computerization. It is increasingly a machineproduced visibility. Now the act of looking is also the act of analysis. Discussing the impact of new imaging technologies, the late digital artist Tristan Humphries noted that:

...digital imaging, with its facility for sophisticated photorealistic fantasies and virtual worlds engages the viewer in an indeterminate struggle between what to believe and what to doubt. The rapid advances in digital technology have not only extended ways of seeing, understanding and representing the world through new imaging technologies, but they have also redefined the ways in which we understand and relate to existing media. ${ }^{7}$

In Beyond visibility: light and dust, I exhibited a seven-panel mural-sized work of inkjet prints on paper. The title of my work, Deep Field (Figure 7), was selected both as an homage and a playful reference to the iconic photograph the Hubble Deep Field (1995). This image is a core sample of the extent of the Universe's observable limits captured by the famous Hubble Space Telescope's Wide Field and Planetary Camera 2 over ten consecutive days.

My work is a speculative and imaginary map of the depths of the night sky at vastly different distances from Earth, incomplete and open to interpretation. As if manipulating a camera lens or a mouse, Deep Field takes the viewer deeper and deeper into space, zooming in and out at different distances from the surface of the work. Like a photographic 'still' frozen in time, it creates an experience of non-visible phenomena in the night sky. These are revealed through fragmentary data, mapped with telescopes and cameras at different wavelengths in the electromagnetic spectrum. The seven panels (somewhat like a series of strip maps) fall

\footnotetext{
${ }^{7}$ Tristan Humphries, 'From the Virtual to the Physical', in Transformations Australia - Digital Imaging and the Art Object, exhibition catalogue, (Melbourne: RMIT University 1998), p. 6.
} 
down the wall and curve out over the floor and back on themselves at their bases. This creates a wave-like structure suggesting the arc of the sky and the curved nature of space and time as we now understand it.

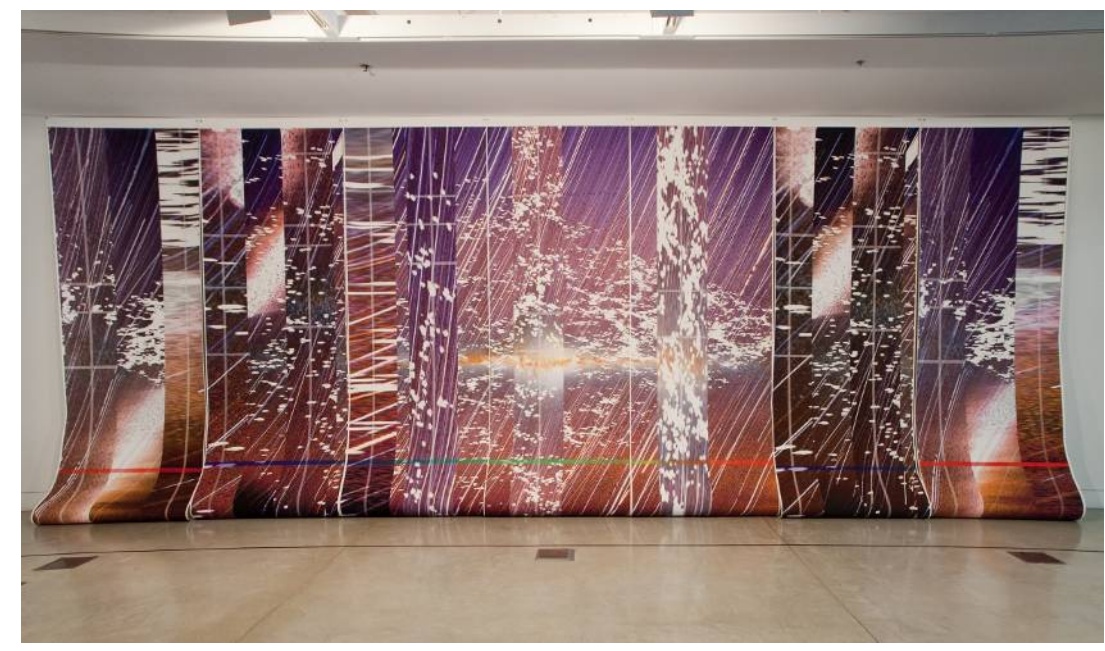

Figure 7. Felicity Spear, Deep Field, 2007, seven pigment inkjet prints, University of Technology Sydney Gallery (Photo Paul Pavlou)

Within the vertical and horizontal structure of Deep Field (Figure 8), everything is seen simultaneously through complex patterns and layers of data, appearing to be at different magnifications and different dimensions. Embedded in this surface are manifestations of coloured light captured in streaks, flashes and pixels, patterns, flecks and dots, exposing the noisy process of image capture and an atomistic, undulating space of foaming and flickering surfaces. A background of time-lapse star-trail images streak across each panel. They were captured in the 1990s by David Malin in his photograph Moonset Over the Warrambungles (MISC 6), in the vicinity of the Anglo-Australian Observatory. These time-lapse images connect our planet to the stars by revealing the rotation of the Earth. This makes the stars follow galactic arcs across the sky and expresses the relationship between time and space in the cosmos.

Across the centre of Deep Field I have inserted a telescopic image of the edge-on view of the Milky Way Galaxy. This image of the Edge-on View of Infrared Milky Way was captured in 2003 by the Two Micron All-Sky Survey (2MASS), using twin infra-red telescopes in Arizona and Chile. Visible in this false-colour, edge-on, inside-out view 
of the Milky Way is a thin disc of stars (the white area), as well as the central bulge or nucleus, surrounded by huge clouds of inter-stellar dust, (the yellow area), which block our view of the galaxy in visible light. Spreading out over much of Deep Field from a suggested apex is a wedge-shaped pattern, reminiscent of the foam formed by waves as they wash up on the beach. It references the Sloan Digital Sky Survey 3-D Universe Map, the wedge-shaped slice of the cosmos detected by the Survey using ground-based, wide-field telescopes in New Mexico. By mapping one quarter of the entire sky the Survey reveals the variations in the distribution of galaxies and the large-scale structure of the sky.
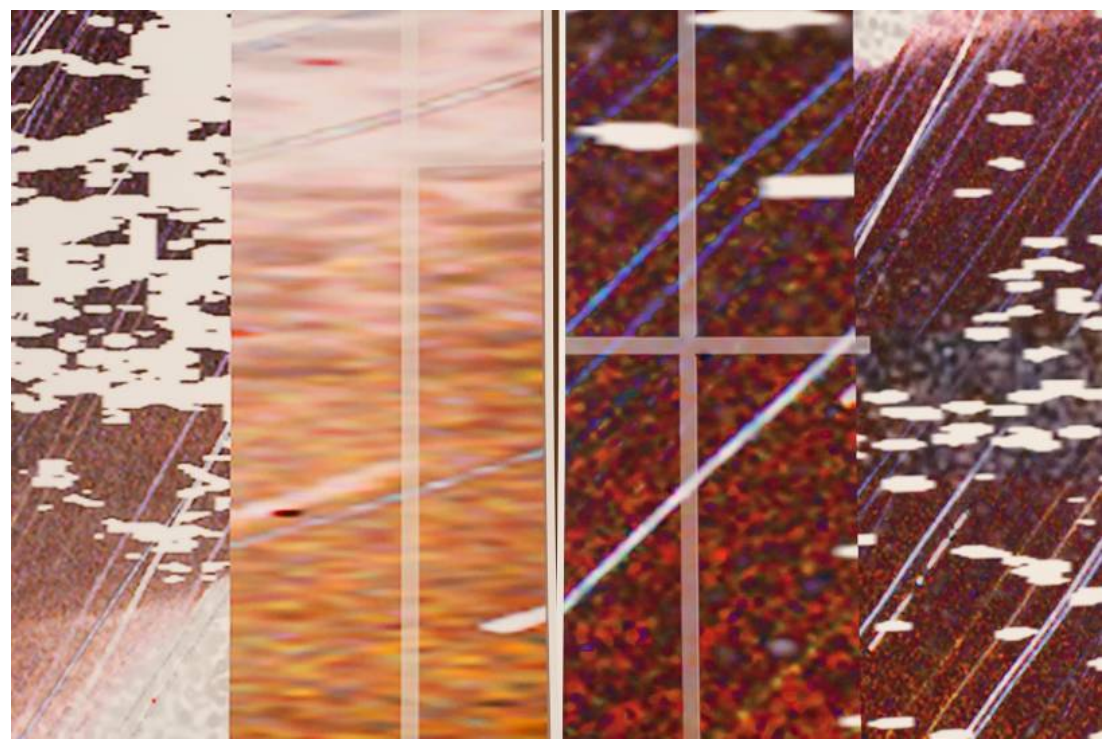

Figure 8. Felicity Spear, Deep Field, 2007 (detail, pigment inkjet prints

As a work of art Deep Field privileges the subjective, the sensual and the aesthetic over clever science, while at the same time immersing the viewer in an experience of the night sky that would not be possible without scientific and technological knowledge and innovation. This speculative map represents parallel phenomena in art and science. Here the structural analogies create another dimension of the real, as well as offering something different from real life. Mapping is always embedded in the subjective conditions of human thinking and is, in the end, the mapmaker's version of reality. While the viewing experience itself may be immediate, it is my hope that the works in this exhibition, Beyond 
Visibility: light and dust remain with the viewer, creating other resonances and leading to further questions.

The natural world is continually being remade on the basis of new understandings. Knowledge of the cosmos is deciphered through the lens of cultural attitudes and beliefs that change over time. Together these exhibitions demonstrate how the mystery of the night sky, and ideas in art and science, are modelled and imagined through continually unfolding and fragmented theories and images. These are analogous to the complex pieces of a puzzle that may never be completed. 